\title{
Analysis of the Experimental Data from MOS Structures in the Case of Large Noise-to-Signal Ratio
}

\author{
L. BorowiCz ${ }^{a}$, P. BorowICZ ${ }^{a, b}$ AND W. RzODKIEWICZ ${ }^{a}$ \\ ${ }^{a}$ Institute of Electron Technology, al. Lotników 32/46, 02-668 Warsaw, Poland \\ ${ }^{b}$ Institute of Physical Chemistry PAS, Kasprzaka 44/52, 01-224 Warsaw, Poland
}

\begin{abstract}
The signal coming from $\mathrm{SiO}_{2}$ layer of MOS structure have large noise-to-signal ratio. This has two reasons first: the dielectric layers have small Raman efficiency, second: the thickness of the dielectric layers are of the order of $10 \mathrm{~nm}$, so the volume of the material irradiated with laser light is small. At the other side spectroscopic and optical data carry the information about important properties of the structure like mechanical stress. Distribution of mechanical stress introduce an important contribution to the electric properties of the electronic systems based on the MOS structures. Therefore, it is important to "distillate" the optical data from the noise. In this contribution the authors discuss some methods of denoising of the Raman signal. The discussed methods compare treatments like wavelet analysis or convolution. The work is illustrated with some examples of the extraction of the data coming from thin layers. The examples of application of the optical data in the description of the properties of the studied structures are presented.
\end{abstract}

PACS numbers: 42.55.Ye, 85.30.Tv, 77.55.-g, 42.60.Mi

\section{Introduction}

The signal coming from $\mathrm{SiO}_{2}$ layer is very small in comparison with scattering coming from Si substrate. There are two reasons for the weakness of the signal: first the efficiency of Raman scattering for $\mathrm{SiO}_{2}$ is very small, second: the amount of irradiated material is small due to thickness of the dielectric layer which is of the order of $10 \mathrm{~nm}$. Also the shape of Raman spectra coming from $\mathrm{SiO}_{2}$ layer is different in comparison with the scattering from silicon. This is due to the structure of dielectric layer that is amorphous in opposite to the crystal structure of silicon. The Raman spectra of $\mathrm{SiO}_{2}$ consist of broad bands in opposite to well defined and sharp line coming from silicon. All these conditions result in that the Raman spectra of $\mathrm{SiO}_{2}$ are difficult to precise interpretation due to not favorable signal-to-noise ratio and to not well defined maxima. It is also very important to improve signal-to-noise ratio without affecting the spectra itself.

The aim of this work was to discuss the mathematical treatment of the Raman spectra that allow to increase the quality of interpretation. The substantial point in the procedure of denoising is confidence level of the mathematical analysis used to improve signal-to-noise ratio.

\section{Experimental}

The MOS structures were manufactured in Division of Silicon Microsystem and Nanostructure Technology.

The Raman scattering was measured with MonoVista Raman microscope (Spectroscopy and Imaging GmbH,
Germany). As a fundamental the visible line of $\mathrm{Ar}^{+}$laser $488 \mathrm{~nm}$ (INOVA 90C FREED) was used. The power of the laser beam on the sample was equal to $\approx 1 \mathrm{~mW}$.

\section{Results and discussion}

The Raman spectra coming from $\mathrm{SiO}_{2}$ layer are very good example of the phenomenon where signal-to-noise ratio makes the detailed analysis unfavorable. This is clearly presented in Fig. 1. Part (a) shows the Raman spectrum of MOS structure. The peak coming from Si substrate has good signal-to-noise ratio. Part (b) of Fig. 1 presents the same spectrum with ordinate that was expanded 175 times in order to present weak bands coming mainly from $\mathrm{SiO}_{2}$ layer. Since all mathematical transformation are done on one-dimensional lists, the abscissa is given in "Number of element" instead of Raman shift directly $\left(\mathrm{cm}^{-1}\right)$.

In order to obtain the spectrum with improved signal-to-noise ratio the one-dimensional list was transformed with wavelet-Fourier transform using the optimal basis set [1]. As a result one gets multi-level lists that consists of 8 sublists of wavelet coefficients. The sublists have the following number of elements: $16,16,32,64,128,256$, 512,1024 . This kind of transformation enables to emphasize the characteristic properties of the spectrum [2]. In Fig. 2 part (a) there are presented wavelet coefficients of all 8 analyzed sublists. It seems that sublists No. 7 and 8 have the coefficients equal to zero. Part (b) of Fig. 2 shows that even 8th sublist has non-zero coefficients with non-random distribution. 


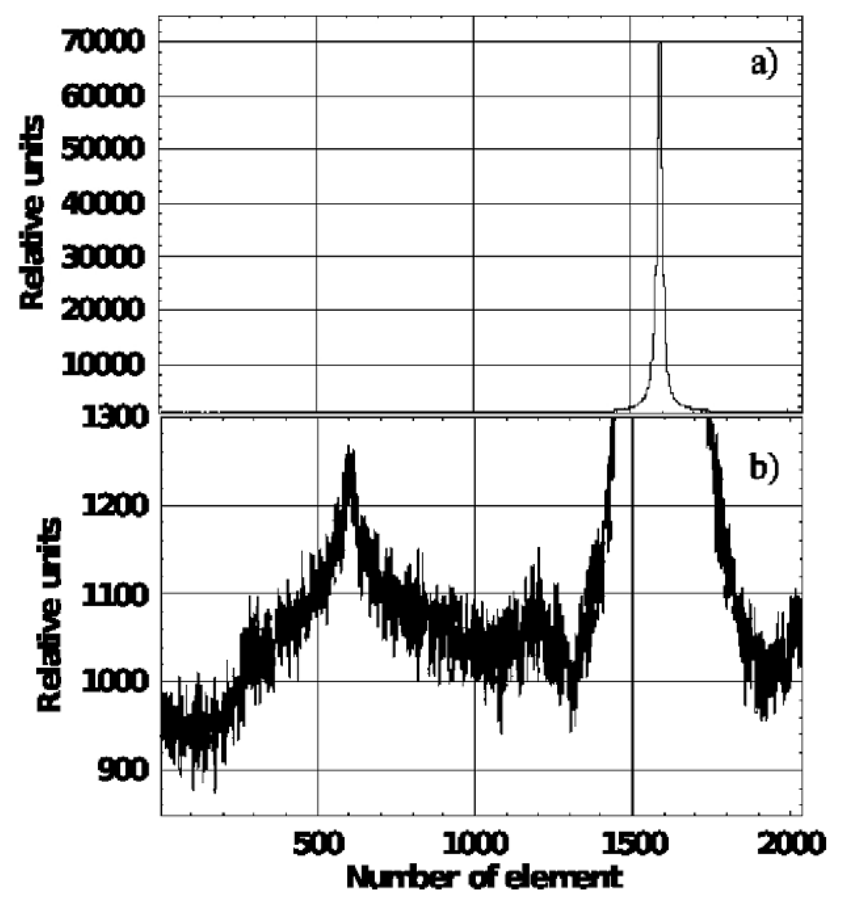

Fig. 1. (a) Raman spectrum of annealed structure $\mathrm{Si} / \mathrm{SiO}_{2} /$ poly-Si $/ \mathrm{Al}+1 \% \mathrm{Cu}$. Strongly dominant peak at the right sight corresponds to the scattering from Si ("520 $\mathrm{cm}^{-1 ")) . ~(b) ~ T h e ~ s a m e ~ s p e c t r u m ~ w i t h ~ a b s c i s s a ~}$ expanded 153 times in order to present weak signals coming from $\mathrm{SiO} 2$ layer.

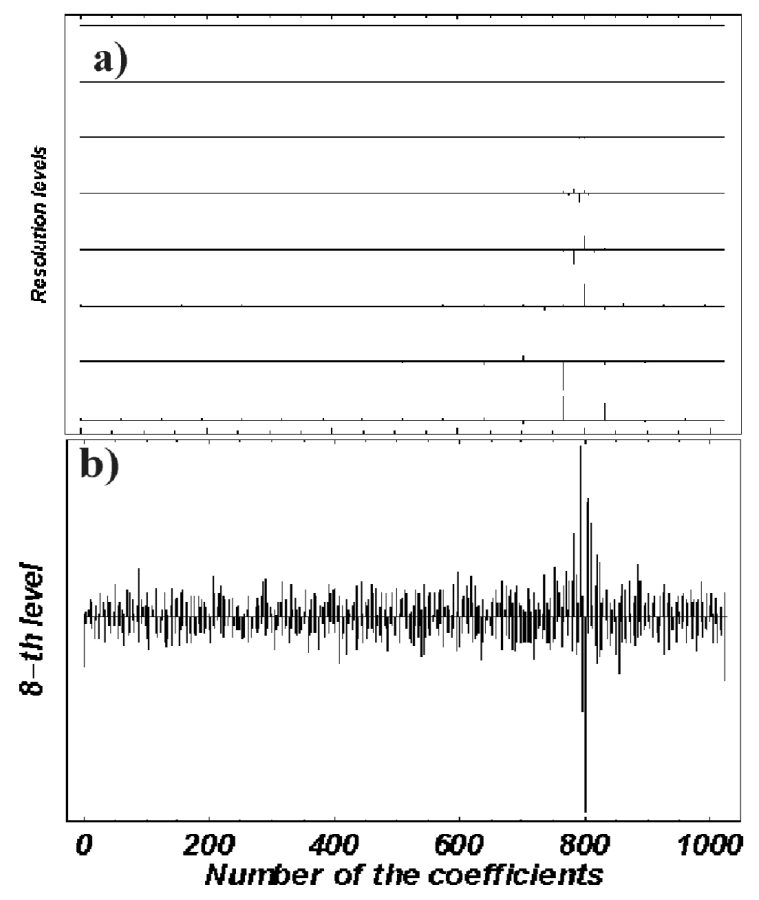

Fig. 2. (a) Wavelet coefficients of analysis of spectrum presented in Fig. 1. The plot presents values of coefficients for all 8 sublists of multilevel list. (b) Coefficients of 8 th sublist presented in the case of expanded abscissa.

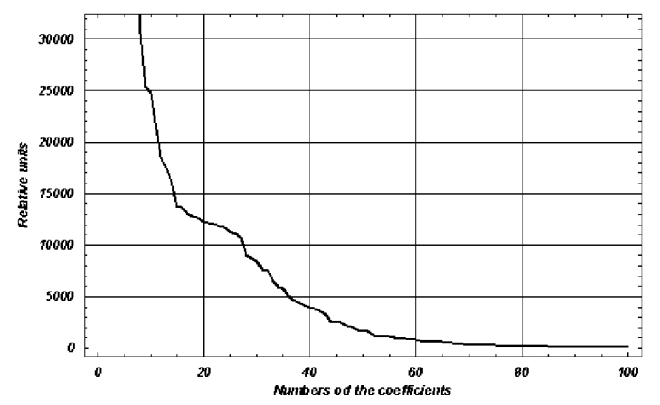

Fig. 3. The value of the wavelet coefficient as a function of the number of coefficient. The data are presented for the 100 largest coefficients.

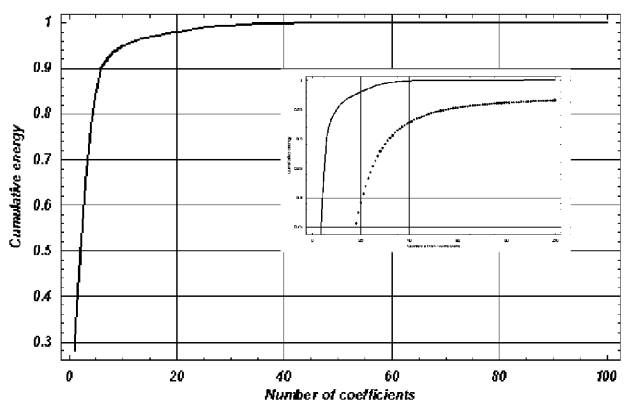

Fig. 4. The value of cumulative energy as a function of number of wavelet coefficients taken into evaluation. The value was normalized to 1 (for details see text). The inset presents the comparison of cumulative energy as a function of number of coefficients for wavelet and FFT analysis.

The important point of the mathematical analysis is: how many coefficients should be taken for the denoising procedure in order to get the optimal performance of the analysis. In Fig. 3 is presented the value of wavelet coefficient as a function of coefficient number for first (largest) 100 coefficients. The values for the coefficient larger than 80 change slow with the coefficient number. The ratio of the hundredth coefficient to the first one is 0.00115. It suggests that the contribution of the information enclosed in hundredth coefficient should be small in comparison to the first one. Let us now come to criteria of the quality of wavelet analysis. In order to evaluate the quality of the denoising the quantity called "cumulative energy" will be used [3]. Cumulative energy is connected with Burgers equation. This equation describes parameters of shock waves and depends on the square of speed. We introduce cumulative energy $E(n)$ in first $n$ wavelet coefficients $c_{i}$ by the following equation:

$$
E(n)=\sum\left|c_{i}\right|^{2} \quad \text { where } \quad\left|c_{i}\right| \geq\left|c_{i+1}\right| .
$$

In Fig. 4 is presented the cumulative energy as dependent on the number of wavelet coefficients. The cumulative energy was normalized to unity. It means that the values of $E(n)$ for $n \leq 100$ were divided by the value calculated for all coefficients. The normalized cumulative 
energy of first hundred coefficients equals to 0.999974. It means that taking first hundred coefficients we preserve the "amount of information" equal to 0.999974 of the total "amount of information" in the Raman spectrum. In order to estimate how many wavelet coefficients should be taken let us have a look on the relative standard deviation of cumulative energy as a function of the amount of coefficients taken for compression*. In order to present the data in the way independent of the number of elements of initial list we introduce the compression factor $k$ equals to ratio of total number of wavelet coefficients to the number of coefficients taken for the compression. The relative standard deviation of the cumulative energy is given by equation

$$
\chi(k)=\left(\frac{\sum\left|\bar{E}_{k}-E_{k}\right|^{2}}{\sum\left|E_{k}\right|^{2}}\right)^{1 / 2},
$$

where $\bar{E}_{k}$ denotes the reconstructed energy and $E_{k}$ the original value. The course $\chi$ as a function of $k$ is presented in Fig. 5. In the case of $k$ values smaller than 20 the increase of $\chi$ with the increase of $k$ is small. In the range of $k$ between 20 and 25 the changes of $\chi$ becomes to be much stronger with increase of the $k$ value. From Fig. 5 it can be deduced that taking first hundred coefficients which corresponds to compression factor equal to 20.5 should be the optimal choice for analyzed spectrum. The comparison of normalized cumulative energy in cases of wavelet analysis and fast Fourier transform (FFT) is presented in the inset in Fig. 4. The abscissa is limited to $0.735 \div 1$ range in order to show the differences between the course obtained for wavelet analysis and FFT. The values of normalized cumulative energy shows that wavelets are much more suitable for denoising procedure than FFT.

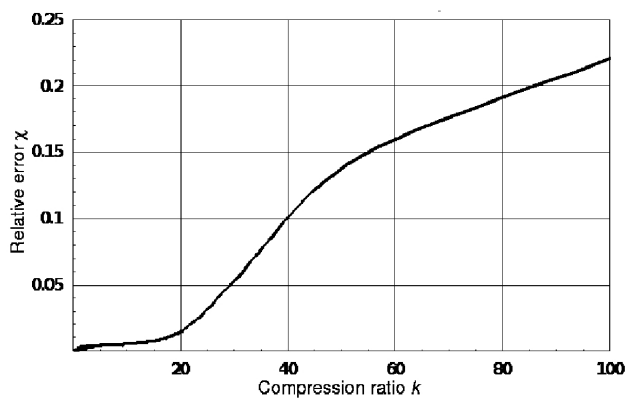

Fig. 5. Relative error of cumulative energy $\chi$ as a function of compression factor $k$.

In Fig. 6a is presented Raman spectrum "reconstructed" after denoising procedure with application of wavelet analysis. The signal-to-noise ratio is much

\footnotetext{
* The analysis presented here has its origin in problems concerning compression of digital data i.e. images with negligible losses of the quality of the compressed data.
}

smaller in comparison with the original spectrum. Part (b) of Fig. 6 presents the part of the spectrum, namely the range of the Raman shift $410 \div 465 \mathrm{~cm}^{-1}$. Spectra presented in Fig. 6 have ordinate converted to the Raman shift after denoising. Vertical grids correspond to position of the Raman bands. Double grids in the ranges between 275 and $280 \mathrm{~cm}^{-1}$ indicate doublets (Fig. 6a). The bands presented in Fig. 6b show maxima for the Raman shift equal to $422.3 \mathrm{~cm}^{-1}, 433.0 \mathrm{~cm}^{-1}, 434.9 \mathrm{~cm}^{-1}$, $450.0 \mathrm{~cm}^{-1}$ and $452.0 \mathrm{~cm}^{-1}$. It seems that the band around $430 \mathrm{~cm}^{-1}$ has more complicated structure than expected $[4,5]$. Since the spectra were recorded with deeply cooled CCD camera (operating temperature $120^{\circ} \mathrm{C}$ ) with long time of accumulation (over $1 \mathrm{~h}$ ) and the distance between found maxima is significantly larger than resolution of the used Raman spectrometer $\left(2 \mathrm{~cm}^{-1}\right.$ vs. $\left.0.2 \mathrm{~cm}^{-1}\right)$ the artificial nature of the maxima rather has to be excluded.
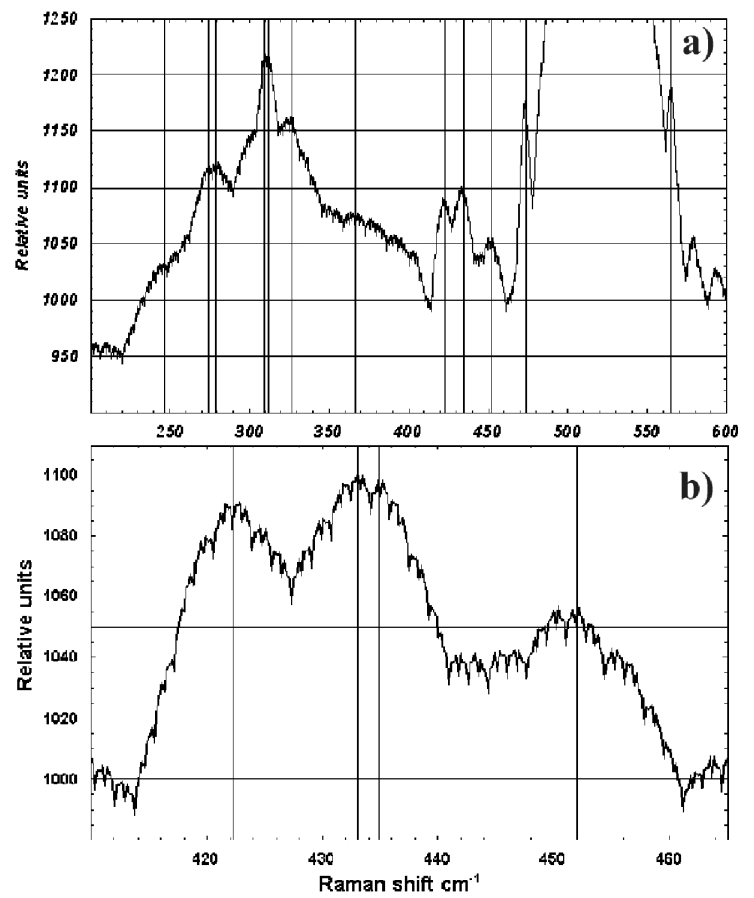

Fig. 6. (a) Reconstruction after denoising with wavelet analysis Raman spectrum presented in Fig. 1. (b) Part of reconstructed spectrum presented in (a). Ordinate expanded in order to present details in the range of $410-465 \mathrm{~cm}^{-1}$.

The band placed between $920 \mathrm{~cm}^{-1}$ and $1050 \mathrm{~cm}^{-1}$ is connected with existence of traces of water in the structure [6]. Annealing at $400{ }^{\circ} \mathrm{C}$ results in escaping of this water combined with even inelastic deformation of material, namely $\mathrm{SiO}_{2}$ layer. Since the escape from the area placed outside of the gate is much easier than the area placed under the gate the influence of the annealing should be much stronger in the case of area that was covered with gate. This is in agreement with microscopic images of the samples where metallic contact and 
poly-silicon gates were etched with HF. Also ellipsometry measurements of both kinds of samples (annealed and non-annealed) agree with the hypothesis of changes of the material upon interaction with water vapor during the process of annealing. The refractive coefficient of $\mathrm{SiO}_{2}$ layer is smaller in the case of annealed sample (in comparison with non-annealed) which is typical of porous media.

\section{Conclusions}

The wavelet analysis essentially improves the signal-to-noise ratio of the Raman signal coming from $\mathrm{SiO}_{2}$ layer in MOS structures. The FFT analysis does not give such good results in denoising as wavelet analysis.

The Raman spectra of $\mathrm{SiO}_{2}$ layer have probably much more complicated structure than expected so far. This is probably due to interaction with water vapor during the process of annealing. The morphology of the silicon oxide layer observed in optical microscopy and ellipsometry measurements support the hypothesis of this interaction.

\section{Acknowledgments}

The author want to thank Mr. Mariusz Latek for his commitment in the experimental part of this work.

\section{References}

[1] R.R. Coifman, M.V. Wickerhauser, IEEE Trans. Inf. Theory 38, 713 (1992).

[2] J.T. Białasiewicz, Wavelets and Approximations, WNT, Warszawa 2000 (in Polish).

[3] S. Wolfram, Mathematica Wavelet Explorer, 1996.

[4] P.F. McMillan, G.H. Wolf, P. Lambert, Phys. Chem. Minerals 19, 71 (1992).

[5] P. McMillan, Am. Mineral. 69, 622 (1984).

[6] P.F. McMillan, J.R. Holloway, Contribut. Mineral. Petrol. 97, 320 (1987). 